\title{
La transformación hacia experiencias expandidas en educación superior: curso \#UAMskills de identidad digital
}

\author{
Transforming to Expanded Experiences in Higher Education: \\ \#UAMskills Digital Identity Course
}

\author{
Javier González-Patiño \\ Universidad Autónoma de Madrid, Madrid, España \\ javier.gonzalezpatino@uam.es \\ Moisès Esteban-Guitart \\ Universitat de Girona, Girona, España \\ moises.esteban@udg.edu
}

\begin{abstract}
Resumen
En este artículo se describe la transformación de un curso de educación superior de la UAM, que durante años se desarrolló de manera presencial, en experiencia de aprendizaje remoto expandido, es decir, mediado por el espacio y cultura digital. La aproximación pedagógica de la personalización educativa-diversificación de recursos, estrategias y actuaciones para facilitar que los aprendices otorguen sentido y valor personal a los aprendizajes - ha sido fundamental para orientar la experiencia aquí se presenta. Se han incorporado actividades de carácter abierto, usando herramientas y plataformas de la web social, que han habilitado a los participantes, constituidos como comunidad de práctica, a crear conocimiento antes, durante y después de las sesiones síncronas, así como, difundirlo en redes, conversar con otros interesados externos y recuperarlo con posterioridad. En las conclusiones se pone de manifiesto cómo articular la diversidad cultural y de trayectorias de participación en el actual escenario social de complejidad e incertidumbre. Se propone la pertinencia de la personalización educativa para facilitar aprendizajes situados y con sentido, desarrollando experiencias expandidas, flexibles y distribuidas en torno a una narrativa transmedia que naturalice la cultura digital en lo que a oportunidades y procesos de aprendizaje se refiere.
\end{abstract}

Palabras clave: Educación superior, aprendizaje conectado, personalización educativa, medios digitales y aprendizaje, comunidad de práctica, trayectorias de aprendizaje, cultura digital

\begin{abstract}
The purpose of this paper is to describe the transformation of a higher education course at the Autonomous University of Madrid from physical teaching to an expanded remote learning experience, mediated by digital culture and space. The pedagogical approach of personalized education - based on providing resources, strategies and actions to facilitate learners to make sense and personal value to learning - has been central in orienting the intervention presented here. Open and connected activities have been incorporated, promoting the use of tools and platforms of the social web which have enabled participants, constituted as a community of practice, to create knowledge before, during and after synchronous sessions, as well as spread it in networks, converse with other external stakeholders and recover it later. The conclusions display how articulating cultural diversity and participatory pathways in the current social scene of complexity and uncertainty. It is considered the relevance of personalized education to facilitate situated and meaningful learning, developing expanded, flexible and distributed experiences around a transmedia narrative that naturalizes digital culture in terms of learning opportunities and processes.
\end{abstract}

Keywords: Higher education, connected learning, personalized education, digital media y learning, community of practice, learning pathways, digital culture

RED. Revista de Educación a Distancia. Núm. 65, Vol. 21. Artíc. 8, 08-01-2021

DOI: https://doi.org/10.6018/red.433881 


\section{Introducción}

La adopción súbita y generalizada de soluciones de "educación remota de emergencia" (Bozkurt y Sharma, 2020, p. 1), como consecuencia de la suspensión de la actividad presencial en las universidades, ha abierto la posibilidad de realizar observaciones y estudios empíricos tomando la circunstancia de remotidad como condición experimental, algo que hubiese sido éticamente imposible de implementar a nivel poblacional en cualquier otro momento.

Sin embargo, más allá de este tipo de estudios observacionales esenciales sería difícil imaginar mejor momento de convergencia mediática, histórico y actual, para aspirar al cambio y mejora educativa a la vez que producimos conocimiento científico. La investigación acción aparece como un enfoque idóneo para un estudio como éste basado en el análisis de una experiencia educativa diseñada para transformar un curso universitario presencial en remoto, pero con una visión de la educación a más largo plazo y para una sociedad hiperconectada (Van Dijck, 2013).

En dicho escenario, los usos de los medios sociales y digitales de comunicación se están transformando con base en nuevos estilos de democratización del aprendizaje y creacióndistribución de contenidos y experiencias (Gee y Esteban-Guitart, 2019; Jenkins, Ford y Green, 2013). Lo que se conoce como cultura participativa y que se basa en la incorporación de la agencia y autonomía de cada comunidad educativa expandida por este empoderamiento digital (Esteban-Guitart y Gee, 2020; Esteban-Guitart, Monreal-Bosch et al., 2020; Jenkins, 2008).

Analizando usos y prácticas tecnológicas relacionadas con el aprendizaje que aparecen en forma de diferentes estilos de mediación semiótica fruto de procesos de actuación participativa-comunicativa (González-Patiño, 2011), observamos complejas transformaciones interactivas de espacios que afectan, entre otros, a la noción de presencialidad.

Desde un punto de vista sistémico, y tomando en consideración el espacio digitalpúblico y privado - medios y tecnologías facilitarían ciertas inclusiones mutuas que serían arrebatadas simbólicamente a este espacio virtual, siempre y cuando las posibilidades y decisiones de los participantes y agentes implicados lo permitan (ideologías educativas, disposiciones legales, convenciones, intimidad, etc.). Aspecto que se traduce tanto en situaciones relacionadas con la enseñanza y el conocimiento, como en otras menos reguladas - como la web social — en las que las personas disfrutan de autonomía para construir y desplegar su identidad social y cultural. Esta condición de convertibilidad simbólica de lo digital que habilita apropiaciones e inclusiones de espacios y actividades antes claramente geolocalizables (trabajo, hogar, facultad, etc.) permite otra peculiaridad propia del trabajo de campo de la presente investigación: constituye el escenario mismo de las prácticas reflexivas y de producción de conocimiento.

De manera complementaria a los estudios que exploran en contextos institucionalizados o reconocibles mediante su localización, este estudio se sitúa en el intersticio de esos lugares, en un ejercicio de aproximación colaborativa, participativa y transformadora que aporta asimismo el corpus de datos. Y precisamente el ánimo de esta publicación es el de documentar para revelar lo que hemos aprendido, haciéndolo comunicable y evaluable, y trascendiendo las soluciones funcionales y el resultado, en un esfuerzo de que el conocimiento se haga visible pero usable, replicable, y por tanto mejorable (Lafuente, Gómez y Freire, 2018).

La transformación hacia experiencias expandidas en educación superior: curso \#UAMskills de identidad digital. Javier González-Patiño y Moisès Esteban-Guitart. 
Otra reflexión, especialmente relevante para el análisis de la experiencia educativa que presentamos en este artículo, atañe a la función de la educación formal superior, pues aunque primordialmente siga siendo la de facilitar la construcción de instrumentos psicológicos que mejoren los proyectos profesionales y de socialización de las personas, no parece tan claro que lo haga tomando en consideración al tipo de aprendiz al que atiende y en consecuencia la interdependencia y sinergias entre las prácticas formales y otras experiencias de aprendizaje cada vez más presentes y difíciles de categorizar (Pereira et al., 2019).

La educación superior en la sociedad conectada tendrá que adaptarse a esta nueva ecología del aprendizaje asumiendo y liderando una transformación que conecte unas prácticas docentes intrínsecamente diversas con las experiencias que ocurren fuera de la institución. Más allá de las facultades de libertad de cátedra y de autonomía universitaria, proponemos asumir como fortaleza, la flexibilidad y la capacidad de adaptación que aportará la diversidad de prácticas docentes que se van a desarrollar como resultado de las diferencias sobre usos relativos a medios digitales y aprendizaje, tanto por parte de los equipos docentes como de las y los estudiantes (recursos disponibles, titulaciones, competencias, destrezas, etc.). Tratar de fijar un marco de soluciones homogéneo, o de buenas prácticas, añadiría rigidez y tensión que podrían provocar un colapso en nuestra capacidad de respuesta, necesariamente adaptativa y ágil, para dar soluciones en un escenario disruptivo sin apenas realizar mejoras estructurales ni disponer de mucho tiempo para hacerlo.

Aunque en ocasiones el debate sobre la mejora educativa como resultado del impacto de lo tecnológico tienda a simplificarse alrededor del aprendizaje y la evaluación (como las cuestiones sobre adaptación y personalización de la enseñanza), un número cada vez mayor de autores y autoras proponen crear áreas de interés que relacionen investigación y creación de conocimiento transdisciplinar, como la de Digital Media and Learning (Gee, 2010) o la aproximación del aprendizaje conectado (Esteban-Guitart, DiGiacomo et al., 2020; Ito et al., 2013) que busca vincular los intereses y las oportunidades laborales y académicas de los jóvenes dentro y fuera del contexto educativo, de modo que nos permitan abordar los desarrollos tecnológicos como situados en una sociedad en profunda y rápida evolución que nos insta a negociar sentidos y significados entre una globalidad cultural, quizá un tanto superficial, y las comunidades de pertenencia más próximas y con profundas implicaciones a nivel de identidad. Un escenario en el que la gestión de la diversidad como fenómeno social predominante pone a prueba buena parte de nuestras categorías y conceptos previos para comprender y diseñar experiencias educativas positivas y óptimas.

Las consideraciones teóricas que compartimos a continuación, antes de presentar la experiencia educativa, resultan relevantes, no solo con la intención de justificarla, sino de dotar de sentido la transformación realizada en una práctica educativa convencional que se venía desarrollando desde hace 6 años en la Universidad Autónoma de Madrid (UAM). La práctica descrita se convierte ahora en una experiencia expandida, en el sentido de mediada por el espacio digital tanto público como privado, con una propuesta de funcionamiento cercano al de una comunidad de práctica (Jovés, Siqués y EstebanGuitart, 2015; Lave y Wenger, 1991) y sobre las bases del aprendizaje conectado (Ito et al., 2013).

En dicho contexto, se considera la diversidad como un requisito para la eficiencia y sostenibilidad, así como se enfatiza el rol activo del aprendiz en tanto que co-diseñador

La transformación hacia experiencias expandidas en educación superior: curso \#UAMskills de identidad digital. Javier González-Patiño y Moisès Esteban-Guitart. 
de la experiencia de enseñanza y aprendizaje (Coll, Esteban-Guitart e Iglesias, 2020; Esteban-Guitart, Monreal-Bosch et al., 2020; Kalantzis y Cope, 2012).

\section{El aprendizaje entendido como creación de conocimiento o desarrollo de competencias en tiempos de la cultura digital}

Según González-Patiño y Esteban-Guitart (2014), cabe considerar una serie de características que identifican la sociedad conectada, también llamada "Sociedad MóvilCéntrica", a saber: la multimodalidad, la convergencia, la creación-difusión, los espacios de afinidad y el conocimiento distribuido. Se trata de elementos que nos obligan resituar la educación formal.

Las prácticas educativas siguen basándose generalmente en la cultura escrita, de hecho, encontramos grandes diferencias y frentes de resistencia a nuevas inclusiones mediáticas por parte de las instituciones y agentes educativos. La rápida expansión de medios audiovisuales, en primer lugar, y la creación del espacio público digital después, marcaron el inicio de un rápido proceso de complejidad semiótica en el que modos y medios se combinaron a través de tecnologías que ofrecían alternativas de comunicación y difusión de elevada diversidad ("Sociedad PC-Céntrica"). En muy poco tiempo, nuestras posibilidades de acceso, creación y negociación de significado se multiplicaron exponencialmente y a esta multimodalidad informacional, todavía habría que añadir la integración o convergencia de dispositivos y plataformas.

Esta noción de convergencia de tipo tecnológico, o de "agnosticismo de dispositivo", pues estamos siempre conectados (allways on) sea cual sea el artefacto que medie, no resulta tan impactante para nuestro análisis como otra que podríamos identificar como convergencia cultural y que tendría que ver con el cambio en los usos de los medios y que para Jenkins (2008) estaría en la base de la cultura participativa propia de nuestra sociedad conectada, o Móvil-Céntrica, según la cual no resultaría sencillo distinguir entre los procesos de creación y difusión de contenido.

La convergencia así entendida tendría que ver con el flujo de contenidos entre diferentes medios y lo que hace la audiencia para disfrutar con aquellas experiencias de entretenimiento que le atraen, propiciando la aparición de nuevas narrativas que se despliegan a través de múltiples medios y plataformas y que conocemos como transmedia (González-Martínez et al., 2019).

Por tanto, lo genuinamente nuevo que estimula el espacio público de internet y sus plataformas, como las redes sociales, es la aparición de una audiencia creativa y participativa, pues publica y difunde y forma parte de comunidades que se articulan identitariamente provocando la formación de fenómenos fan o fandoms, cuyas prácticas no consisten en una mera sumisión a la persuasión de la industria mediática, sino un diálogo con sus textos culturales mediante relatos que aparecen interrelacionados manteniendo, a su vez, independencia narrativa y sentido completo (Lacasa, 2018; Lacasa, de la Fuente y Méndez, 2020). De este modo, los diferentes soportes digitales facilitan la creación y crecimiento de espacios de afinidad o comunidades líquidas de interés (Gee y Esteban-Guitart, 2019), que con frecuencia se organizan en sitios web que permiten pertenecer y abandonar con rapidez y donde se comparten recursos y valores mientras los grupos se forman y se transforman sin cesar (Lacasa et al., 2017). En

La transformación hacia experiencias expandidas en educación superior: curso \#UAMskills de identidad digital. Javier González-Patiño y Moisès Esteban-Guitart.

Página - 4 - de 24 
definitiva, internet ha cambiado el sentido de la creación y localización del conocimiento, que ahora es distribuido y tiene lugar en una ecología que cambia con el tiempo. Como proponen Gee (2013) y Jenkins (2008), en la sociedad de la cultura digital irrumpe una nueva "mente o identidad social" resultado de la unión entre la colaboración distribuida y los artefactos culturales que ha producido.

\section{La necesidad de producir mejoras y nuevas experiencias educativas para la sociedad conectada: la personalización educativa}

Aunque como indicábamos la función principal de la educación superior formal permanece, con una intención explícita y sistemática que se concreta en un currículum único y homogéneo, es necesaria e inaplazable una profunda reflexión de, al menos, el modelo de aprendiz al que se dirige y el sentido mismo de la institución, así como la articulación y conexión de las prácticas educativas formales frente a otras experiencias de aprendizaje. La educación es un invento social (Bruner y Palacios, 2008), por tanto, mostrará diferentes grados de desajuste con la sociedad que la convive, pues esta se transforma y exige a aquella, adaptarse sin cesar. La educación formal, en las condiciones de la sociedad conectada, debe tener presentes las coordenadas de esta nueva ecología del aprendizaje (Coll, 2013); así como la conexión de las prácticas docentes con las experiencias que ocurren fuera de la institución. Convendría pues, situar dicha actuación educativa dentro de un marco teórico pedagógico que además de justificarla, sirva de orientación para dotarla de sentido. Este es, precisamente, el objetivo principal del presente artículo, a saber: describir y analizar en profundidad un estudio de caso que nos permita identificar los principios y elementos centrales de la personalización educativa como aproximación ajustada para una educación propia de la actual sociedad conectada.

Comenzaremos con una precisión terminológica para distinguir la personalización educativa de otras concepciones frecuentemente utilizadas como la de aprendizaje personalizado o personalized learning (Chen, 2008; Grant y Basye, 2014; Minguillón et al., 2005). Para nosotros, la personalización educativa consiste en un medio, no el resultado de un proceso. Es decir, el aprendiz es el que da sentido a las distintas experiencias de aprendizaje, de modo que mientras la personalización integra actuaciones en el plano interpsicológico o social; el aprendizaje, como proceso de desarrollo de competencias oconstrucción de conocimiento, lo haría en el plano intrapsicológico o personal (Esteban-Guitart y Gee, 2020).

Por tanto, la personalización educativa incluiría esa globalidad diversa de estrategias, actividades y recursos que proporcionamos para que las y los estudiantes logren aprendizajes con sentido y valor personal, y no solo una "customización" del proceso de aprendizaje o una individualización de este (Penuel y Johnson, 2016; Pérez Guerrero y Ahedo Ruiz, 2020). Según lo definen Coll, Esteban-Guitart e Iglesias (2020, p. 35): "La personalización (...) es aquella actividad, práctica o experiencia que vincula los intereses, objetivos y opciones de los aprendices con los objetivos y actividades educativas a partir de su participación-implicación en el control-elección de los procesos implicados (qué, como y cuando aprender)".

El aprendizaje con sentido y valor personal, independientemente de su grado de formalización, se fundamentaría en aquellas experiencias con impacto cognitivo y

La transformación hacia experiencias expandidas en educación superior: curso \#UAMskills de identidad digital. Javier González-Patiño y Moisès Esteban-Guitart. 
emocional que propician en el aprendiz ampliar conocimientos sobre su historia personal y la realidad en que vive, ofreciéndole mejores condiciones para intervenir en ella o crear planes de acción futura (Coll, 2018). En la Figura 1 se muestran los componentes o dimensiones del proceso interrelacionado sobre el que se desarrolla la personalización educativa con el fin de ofrecer una herramienta conceptual para analizar su funcionamiento.

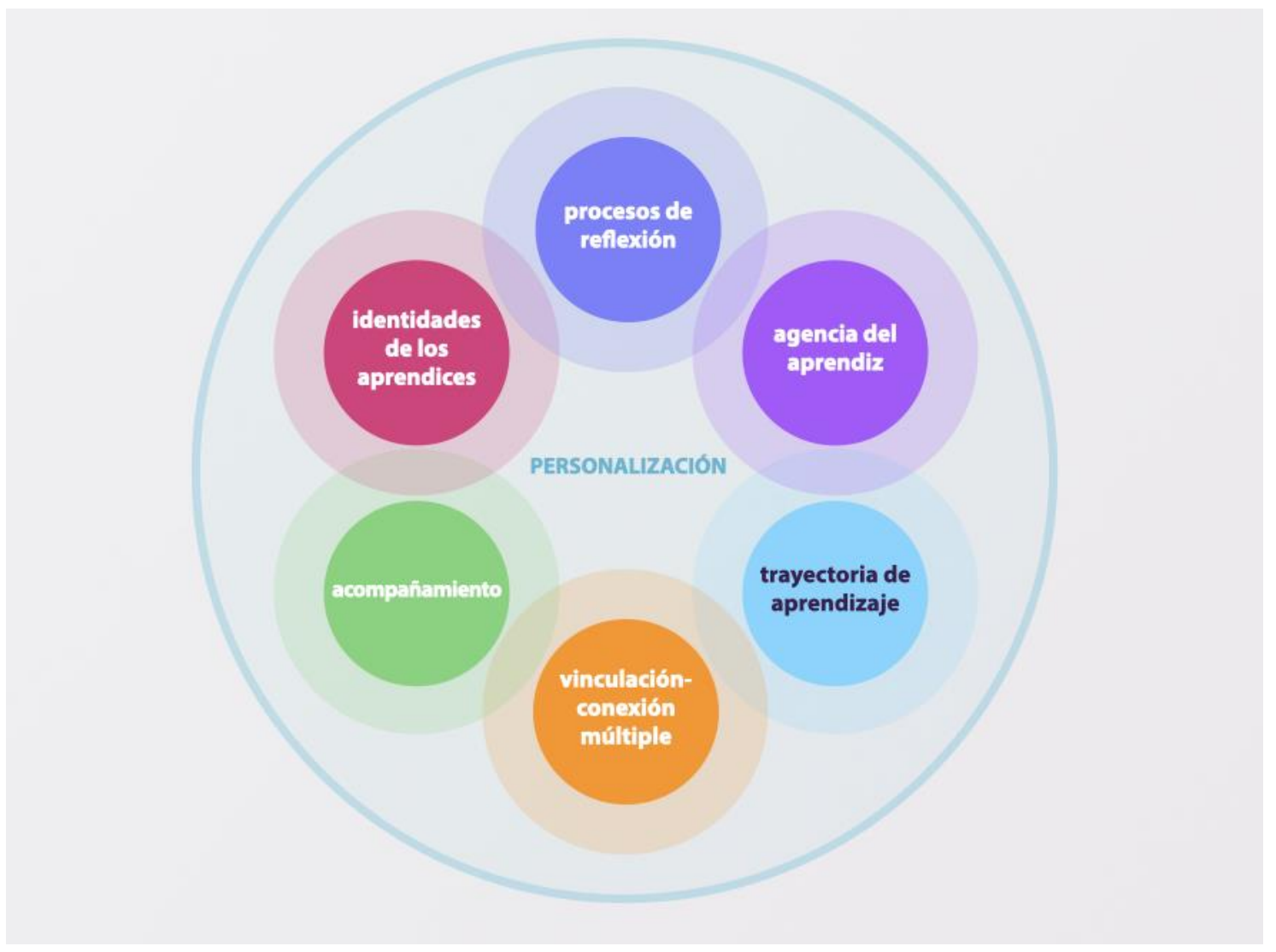

Figura 1. Dimensiones de la personalización educativa Fuente: Elaboración propia

La personalización necesariamente toma como punto de partida las identidades de las y los aprendices, lo que implica indagar y descubrir aquello que perciben como significativo o esencial, bien sean intereses, conocimientos previos, actividades, instituciones, personas o artefactos culturales. Se atendería, asimismo, a la dimensión proyectiva de la identidad en forma de proyectos de vida y decisiones. La identidad así entendida consiste en un proceso de resignificación continuo sensible al impacto de actividades, lugares, instituciones, personas y objetos percibidos como significativos por parte del que aprende (Esteban-Guitart, 2016, 2019). Optamos por la noción de identidad frente a la de interés, por lo general asociada a la personalización del aprendizaje (Walkington y Hayata, 2017), pues la primera engloba a la segunda. La identidad se vincula al ámbito subjetivo del sentido del aprendiz (Esteban-Guitart, 2016; Esteban-Guitart y Moll, 2014) mientras los intereses pueden reducirse a aspectos más superficiales, como preferencias o gustos personales (González-Patiño y Esteban-Guitart, 2019).

Las identidades de los estudiantes son concebidas como el inicio de un proceso pedagógico en constante transformación mediante andamiaje, es decir, vinculando

La transformación hacia experiencias expandidas en educación superior: curso \#UAMskills de identidad digital. Javier González-Patiño y Moisès Esteban-Guitart. 
elementos identitarios con contenidos, competencias u objetos de aprendizaje, las identidades se van configurando y desarrollando. Con el objetivo de identificarlos, se ha propuesto un recurso basado en la creación de "artefactos identitarios", producciones realizadas por el propio aprendiz a través de las cuales proyectan sus intereses, aficiones, actividades, personas, objetos y espacios percibidos como especialmente significativos, que posteriormente serán utilizados por las y los profesores con finalidades curriculares y pedagógicas (Esteban-Guitart, 2019). Numerosos estudios concluyen que los aprendizajes más resilientes, con un mayor impacto en la memoria a largo plazo, son aquellos que parten y se conectan con las necesidades, intereses y objetivos de los aprendices (Barron, 2006; Staus et al., 2020).

Los procesos de reflexión, personal y grupal, además de profundizar en la identificación de dichos intereses e identidades permiten enriquecerlos, haciéndolos explícitos, y también sirven para analizar su origen y desarrollo, así como las consecuencias que implican, independientemente de si este interés u objetivo-objeto identitario ha surgido en el contexto formal o en cualquier otro. En definitiva, los intereses, identidades y procesos de desarrollo y aprendizaje de los estudiantes son resultado de procesos dialógicos de discusión-reflexión (Oliveira y Coelho, 2020). De entre los recursos que se suelen utilizar para propiciar la reflexión encontramos desde diarios de aprendizaje significativo hasta cartografías de espacios y trayectorias de aprendizaje (Esteban-Guitart et al., 2017).

El siguiente componente sobre el que se desarrolla la personalización son los procesos de agencia y que hacen referencia a la participación y el control del aprendiz sobre sus propios aprendizajes, en particular en la definición de objetivos, el co-diseño de actividades y la evaluación. Existe evidencia empírica sobre la relación entre la participación de las y los estudiantes y su implicación, con las mejoras de rendimiento académico (Perin, 2011). Para favorecer la agencia del aprendiz se le suele implicar en la creación de planes personales que permiten desarrollar trayectorias de aprendizaje individuales. A pesar de que la educación formal convencional atiende a un modelo homogéneo de educación (Coll, 2013), hace tiempo que se están estudiando trayectorias profesionalizantes que permiten credenciales apilables, secuencias compuestas por numerosos programas muy enfocados y de corta duración (stackable learning), para promover el logro y la movilidad ascendente (Giani y Fox, 2017), o el creciente interés sobre la diversidad de lugares, agentes, servicios y situaciones de aprendizaje que trascienden los espacios y tiempos educativos tradicionales (Álvarez-Arregui y Arreguit, 2019; Barron, 2006; Esteban-Guitart et al., 2018; Nasir et al., 2020). La condición forzosa de educación remota sobrevenida de la Covid-19 ha puesto de manifiesto la ingente variedad de procesos de aprendizaje en el ámbito informal que a través de los medios digitales y la web social (herramientas, contenidos, plataformas, comunidades y redes de afinidad) permiten que las personas de manera activa puedan desarrollar competencias y adquirir habilidades necesarias para afrontar retos importantes para su vida personal y profesional (Gee y Esteban-Guitart, 2019; González-Patiño y Esteban-Guitart, 2014).

Por otra parte, la vinculación-conexión múltiple o bimodal, que aparecen en la Figura 1, se refiere a la vehiculación de experiencias de aprendizaje. Aunque tengamos constancia de que los aprendizajes tienen lugar a lo largo y ancho de la vida (Esteban-Guitart et al., 2018), esto no implica de suyo que las experiencias, contextos o actividades estén conectadas entre sí, al contrario, es necesario diseñar intervenciones de vinculaciónconexión explícita que conecten experiencias de aprendizaje de los aprendices, a nivel psicológico-individual, así como agentes, tiempos y espacios educativos, como por

La transformación hacia experiencias expandidas en educación superior: curso \#UAMskills de identidad digital. Javier González-Patiño y Moisès Esteban-Guitart.

Página - 7 - de 24 
ejemplo, cuando se vinculan centros educativos, familias y entidades comunitarias (Rodríguez-Ruiz et al., 2019). Hablamos entonces de vinculación-conexión múltiple o bimodal porque la vinculación es doble (Bronfenbrenner, 1985): a nivel micro se articulan experiencias de aprendizaje, conocimientos y experiencias previas con otras nuevas (vinculación psicológica) y, por otro lado, se establecen a nivel meso relaciones de continuidad educativa entre distintos contextos de vida y socialización mejorando su potencial evolutivo o de aprendizaje (vinculación comunitaria). Cabe recordar, según Bronfenbrenner (1987, p. 58), que la eficacia e impacto de un mesosistema aumenta en la medida que: "los roles, actividades y díadas en las que participa la persona vinculante con otros entornos estimula la aparición de confianza mutua, una orientación positiva, el consenso de metas entre entornos, y un equilibrio de poderes progresivo que responda a la acción en nombre de la persona en desarrollo".

Para que estas conexiones puedan llegar a darse es necesario que se cumplan ciertas condiciones sociales, materiales y otras relativas a los intereses y objetivos del que aprende y que subyacen a trayectorias de aprendizaje que las movilizan (DiGiacomo et al., 2018). El acompañamiento sería por tanto aquel componente esencial del proceso para que a partir de las expectativas y necesidades del aprendiz pueda disponer de las experiencias educativas que precisa en cada momento. En este sentido, implicaría tanto reconocimiento y ayuda pedagógica y social como condiciones materiales que permitan la vinculación de identidades, objetivos y proyectos de los aprendices con oportunidades, servicios, agentes y recursos sociales y educativos.

El análisis que presentamos en este artículo sobre una experiencia expandida, a través de la web social, forma parte de una iniciativa más amplia de investigación acción, que excede a los propósitos de esta publicación, y que pretende reflexionar sobre los fundamentos del aprendizaje conectado permitiéndonos, además, incorporar los componentes de la personalización educativa identificados y descritos anteriormente. Para concretarlo, se presenta una aproximación operativa y aplicada de la transformación realizada sobre un curso de identidad digital de la UAM que hasta el confinamiento obligado consistía en una práctica educativa presencial de tipo convencional. De modo que a sobremanera, la experiencia nos permite ilustrar teórica y prácticamente procesos de transición de la docencia física a la virtual.

\section{Transformando el curso \#UAMskills de identidad digital en una experiencia expandida.}

La experiencia de aprendizaje que se describe e ilustra en esta publicación es una actividad participativa creada a partir de la necesidad de transformar un curso presencial en remoto, pero que yendo más allá de la emergencia educativa se planteó con el propósito de convertirlo en una experiencia conectada y que reúna las condiciones que se esperan para la educación de la cultura digital y sobre las que hemos reflexionado previamente: modular y apilable (stackability), con sentido para el aprendiz, diversa y personalizable, flexible y ubicua, participativa y basada en comunidades, híbrida e incorporando una narrativa transmedia, orientada tanto al desarrollo de competencias como a la adquisición de habilidades y que incorpore la evaluación de logros, procesos y aplicabilidad de los aprendizajes (accountability).

Resulta especialmente relevante destacar la dimensión participativa que motiva esta transformación. Hemos tratado de "cultivar" una comunidad alrededor del interés de las y los participantes en la mejora de su proyección profesional desde un entorno educativo formal, y aun así, hemos planteado una actividad de aprendizaje eminentemente situado

La transformación hacia experiencias expandidas en educación superior: curso \#UAMskills de identidad digital. Javier González-Patiño y Moisès Esteban-Guitart.

Página - 8 - de 24 
legitimándola a través de dinámicas identitarias y de participación periférica propias de las comunidades de práctica (Lave y Wenger, 1991; Wenger, 2005; Wenger et al., 2002). Ello permite favorecer relaciones entre contextos no formales e informales, como la incorporación del programa para educación superior de una de las plataformas de social media líderes en el ámbito profesional (Hootsuite's Higher Education Program). No obstante, la difusión y alcance que las nociones de cultura participativa y comunidad de práctica han logrado de un tiempo a esta parte en debates académicos y profesionales, y particularmente en la investigación educativa, deben animarnos a revisar críticamente cómo ha afectado este éxito a los valores centrales que animaron los primeros años de la teoría del aprendizaje situado y la cultura participativa. Es decir, cuando su foco se centraba en comunidades profesionales relativamente definidas, cara a cara, preguntándonos qué significa ahora aplicar estas ideas sobre el aprendizaje y la participación en la universidad, las comunidades online o en equipos de trabajo en las empresas (Jenkins et al., 2015).

\subsection{Contexto del proceso de transformación hacia la personalización Educativa}

El curso en el que se centra este artículo, "Construye tu presencia online y medios sociales como profesional", forma parte desde hace 6 años de la oferta formativa de la Oficina de Prácticas Externas y Empleabilidad (OPE). Se trata de un servicio de la UAM encargado de implementar la empleabilidad de toda la comunidad universitaria y promover el desarrollo laboral de los y las estudiantes y titulados/as. La OPE gestiona un buen número de cursos apilables de corta duración en competencias profesionales y áreas curriculares transversales con reconocimiento de créditos ECTS (credencialización modular) a través del Centro de Formación Continua de la UAM bajo las denominaciones UAMempleabilidad o UAMskills: comunicación y trabajo en equipo, Design Thinking, liderazgo, negociación integradora, presentar en público y cómo afrontar estrés laboral y burnout, entre otros. Concretamente, el curso sobre identidad digital pertenece al programa avanzado dirigido a estudiantes de todas las titulaciones de tercer curso de grado en adelante, posgrados y miembros de Alumni UAM. Estos cursos siempre están dirigidos por personal docente e investigador de la universidad, aunque pueden y suelen participar profesionales invitados por la o el director de cada curso.

El primer autor de este artículo es el director del curso, además de responsable de Mediática, accesible en http://mediatica.co (González-Patiño, 2012), colectivo que realiza labores de asesoramiento participativo a comunidades que desean producir cambios en sus usos y prácticas digitales desde la perspectiva de la personalización educativa. El objetivo que nos propusimos fue aprovechar el reto de adaptar, en poco tiempo, un curso universitario de corta duración al contexto de educación remota forzosa, para experimentar e investigar sobre el impacto del componente expandido, la mediación de tecnologías digitales y herramientas de la web social, sobre un formato que venía apoyándose desde hace años en la presencialidad con buenos resultados, como reflejaban las encuestas a las y los estudiantes.

El trabajo de implementación de esta experiencia que se describe en este estudio se ha extendido desde de marzo hasta mediados de mayo de 2020. La riqueza estructural de la participación ha permitido conseguir un modalmente diversificado corpus de productos que facilita un buen número de abordajes analíticos posibles, que no se abordan en esta publicación, pero que permiten ilustrar procesos de investigación y análisis visual (González-Patiño, 2011; González-Patiño y Esteban-Guitart, 2015; Lacasa, de la Fuente

La transformación hacia experiencias expandidas en educación superior: curso \#UAMskills de identidad digital. Javier González-Patiño y Moisès Esteban-Guitart. 
y Méndez, 2020; Mitchell, 2011; Pink, 2013; Thomson, 2010). En particular, supone un ejercicio de etnografía del espacio público y privado digital, basado en la observación participante en las sesiones síncronas y conversaciones informales con estudiantes y otros agentes implicados, como el personal de OPE UAM. Asimismo, se recogieron datos de diversas fuentes complementarias como la valoración de calidad docente, observaciones informales, documentación y mantenimiento del sistema de gestión de aprendizaje o la lista de correo.

\subsection{Procedimientos, protocolos y escenarios para la colaboración distribuida}

Antes de empezar el curso, las y los inscritos recibieron una guía de participación en la que se presentaba con suficiente detalle el tipo de experiencia de aprendizaje del que iban a formar parte, con la intención de que generaran expectativas diferentes a las que ya tenían sobre docencia universitaria más canónica y de referencias de anteriores ediciones del curso:

Cada vez dedicamos más tiempo a internet, personal y de trabajo, sin embargo, esto no nos vuelve necesariamente más competentes a la hora de utilizar las plataformas y redes sociales como herramientas profesionales. El objetivo principal de este laboratorio es que cada participante desarrolle habilidades y competencias digitales básicas pero suficientes, para que cuando lo finalice haya diseñado y desarrollado su presencia online y en medios sociales de manera que le permita mostrarse y establecer relaciones con profesionales o instituciones relevantes para sus intereses académicos o laborales. Se trata de una experiencia de aprendizaje caracterizada por las trazas de lo que reconocemos como cultura digital (conectada, abierta, activa, participativa, etc.) y con cierto énfasis en aprender haciendo y metodología por proyecto... de hecho, el más importante que tendrá cada participante en toda su vida... juno mismo!

Extracto 1. Texto de presentación en la guía de participación del curso

El cronograma del curso sobre reputación online \#UAMskills que aparece en la Figura 2 también está incluido en la guía de participación. Se puede apreciar que su diseño de contenido está estructurado alrededor de cuatro áreas de interés:

- Cómo seleccionar plataformas y redes sociales para construir tu presencia online y establecer una red de contactos que mejore la empleabilidad: redes generalistas y verticales (especializadas), localizar contactos relevantes, compartir contenido, etc.

- Publicar con herramientas abiertas de gestión de contenido (WordPress, Blogger, etc.): crea un espacio o sitio web como hub de tu presencia online.

- Hacer red, diseñar la guía de estilo personal, conectar las distintas plataformas y perfiles, y conseguir un determinado número de contactos cualificados (visibilidad).

- Medir y analizar, iniciación en herramientas e indicadores que permiten evaluar la difusión e influencia (SEO, SEM, engagement, Google Analytics, etc.).

La transformación hacia experiencias expandidas en educación superior: curso \#UAMskills de identidad digital. Javier González-Patiño y Moisès Esteban-Guitart. 


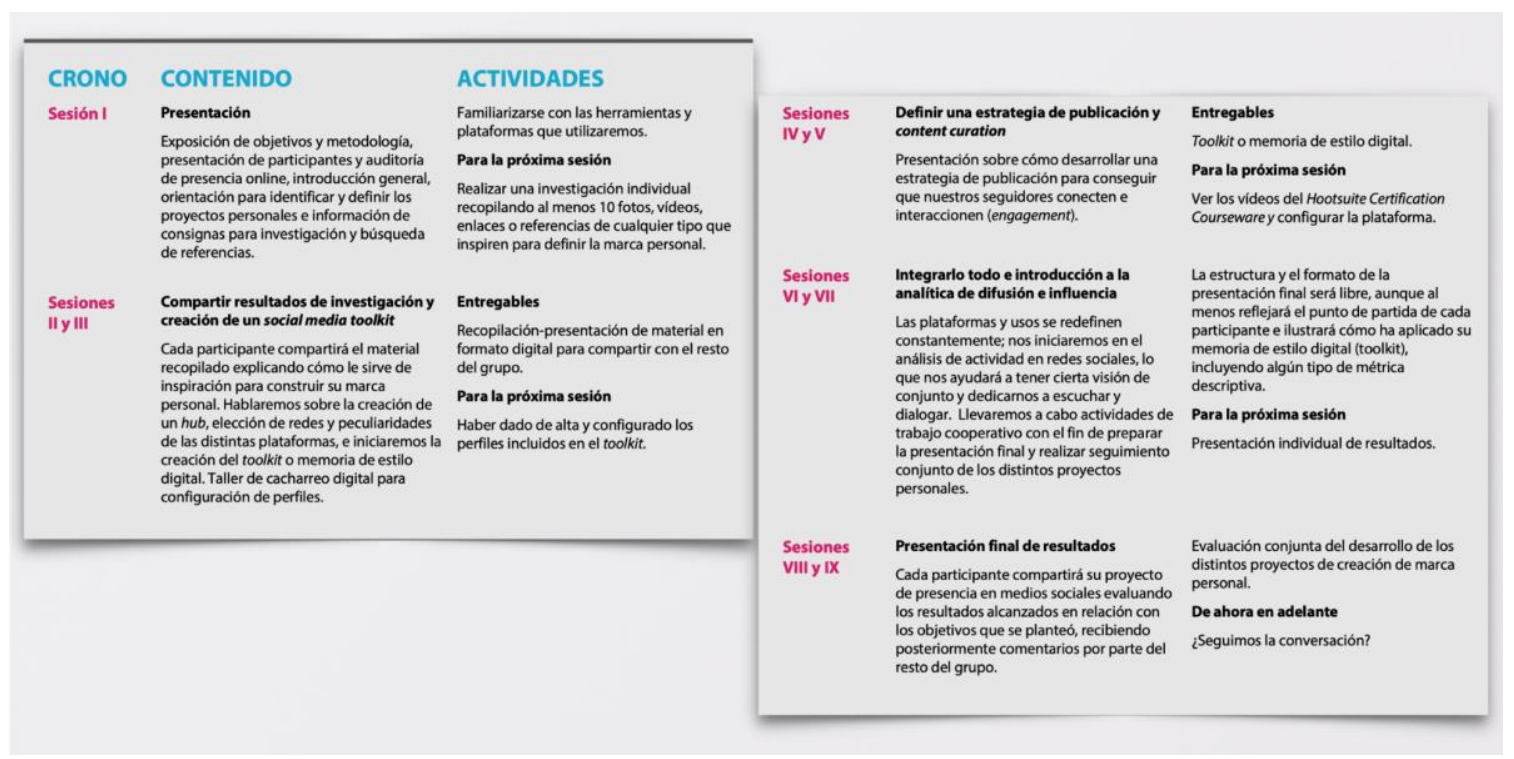

Figura 2. Cronograma del curso incluido en la guía de participación

Sin embargo, como se puede comprobar analizando la Figura 2, esta estructura no prevalece en la organización de las sesiones síncronas en remoto que lejos de seguir la distribución de clases respetando el formato convencional de temas, se integran en una propuesta que deliberadamente mezcla contenido y actividades con el objetivo de ofrecer una narrativa centrada en la misión de cada participante de crear su posicionamiento o identidad en el espacio de internet y las redes sociales. Las sesiones síncronas se realizaron mediante videoconferencia y comprendían tiempo tanto para ampliar la base de conocimientos sobre el contenido a tratar como para la puesta en común de los avances del trabajo personal de cada participante sobre el que recibían retroalimentación del grupo (director del curso y resto de los participantes). La distribución temporal era negociada en cada sesión y, de hecho, compartir esta confluencia de intereses resultó ser un elemento de cohesión muy apreciado por la comunidad que se estaba formando. Finalmente, las presentaciones que se utilizaron estaban a disposición del grupo en el repositorio en la nube junto a recursos extra que aportaron conjuntamente el profesor y el resto de miembros. Asimismo, los participantes se beneficiaron de acceso al Hootsuite's Higher Education Program, que incluye por un lado la posibilidad de aprender y utilizar las características avanzadas de una de las plataformas de soluciones para social media líderes en el ámbito profesional, Hootsuite Pro, y por otro, acceso a su programa formativo superior con certificación de reconocido prestigio internacional, Hootsuite University.

Una cuestión central en esta experiencia pedagógica universitaria en tiempos de pandemia es analizar la mediación y apropiaciones que los participantes hacen del espacio digital de internet, en el que como comunidad que aprende se trata de estar presente y participar con un discurso transmedia que les identifique como actores globales y plantee unos protocolos comunes, creando canales que promuevan la interacción y la producción colectiva y abierta de conocimiento y permitan procesar la información relevante. La estrategia para conseguir una propuesta experiencial, multimodal y expandida de estas características, un curso que se proyecta por encima de lo sincrónico en remoto, lo próximo o interno al grupo, y lo temporal, y que apuesta por la producción colectiva de

La transformación hacia experiencias expandidas en educación superior: curso \#UAMskills de identidad digital. Javier González-Patiño y Moisès Esteban-Guitart. 
conocimiento público, consistió en diseñar, implementar y utilizar los siguientes elementos funcionales:

- El nodo central o hub que integra la participación y la comunicación, construida mediante un sistema de gestión de aprendizaje, Learning Management System o LMS, (Google Classroom dentro del sistema GSuite para Educación del colectivo Mediática) que sirve para gestionar de manera convergente la información y contenidos, actividades, y seguimiento, así como para integrar y dar soporte a las acciones en redes sociales y otras funcionalidades que se han ido incorporando tanto para la celebración de las sesiones síncronas como para la dinamización de la comunidad (lista de correo en, videoconferencias, Hootsuite's Higher Education Program, etc.).

- Un breve manual o guía de estilo incluido en la guía de participación del curso que aporta el marco de protocolos comunes deseables para la comunidad, con sugerencias y recomendaciones técnicas y conceptuales sencillas para mejorar la calidad de la participación.

- Redes sociales para invitar a la conversación dinamizando aportaciones y discusiones, y para generar confianza aumentando las posibilidades de relación y acceso entre los participantes y entre éstos y posibles interesados, como ocurrió con ciertos estudiantes y profesionales que asistieron a anteriores ediciones del curso (ver Figura 3). En la guía de estilo digital se proponía la utilización de las etiquetas o hashtags \#reputaciononline \#uamskills o mencionar ciertas cuentas institucionales como @UAM_Madrid, @OPEUAM y @_mediatica para alcanzar esos objetivos. Asimismo, desde la dirección-mediación académica se utilizaron las cuentas de Mediática en distintas plataformas que permiten adecuarse a cada objetivo de manera diferente: Twitter (https://twitter.com/_mediatica) y Facebook (https://www.facebook.com/mediatica.co) para promover la conversación y micropublicaciones, YouTube (https://www.youtube.com/mediaticaco) para contenido audiovisual, la red de networking profesional LinkedIn (https://www.linkedin.com/company/mediática) para dinamizar la discusión, entre muchas otras (Instagram, Slideshare, Researchgate.net, etc.).

- La producción de las sesiones síncronas en remoto se realizó recurriendo a la funcionalidad Google Meet incluida en Gsuite, que además de ofrecer la posibilidad de crear emisiones de tipo streaming con invitados por videoconferencia, se integra y facilita el flujo de trabajo para el almacenamiento y acceso posterior al contenido audiovisual en YouTube.

- Para la gestión de trabajo colaborativo online y la lista de correo, una herramienta de comunicación fundamental para esta comunidad, se utilizó asimismo la plataforma G Suite para educación de Mediática (Calendario, Grupos, Drive, Documentos, Presentaciones, Hojas de cálculo, etc.) que facilitaba la integración sin demasiado esfuerzo con el LMS Classroom.

- También se han tenido presentes cuestiones relativas a diseño adaptable, responsive design, para incorporar las posibilidades que ofrece el acceso generalizado a través de móviles, y a diseño accesible para facilitar contenidos y procesos a personas con diversidad funcional; ambas cuestiones fueron otro de los factores que influyeron en la adopción del sistema Gsuite de Google, de tal manera que, por ejemplo, el hub se construyó con Classroom que es una plataforma con aplicaciones y acceso web optimizado para cualquier tipo de dispositivo (móviles,

La transformación hacia experiencias expandidas en educación superior: curso \#UAMskills de identidad digital. Javier González-Patiño y Moisès Esteban-Guitart. 
tabletas y ordenador) y la funcionalidad de videoconferencia Meet permite generar subtítulos en directo.

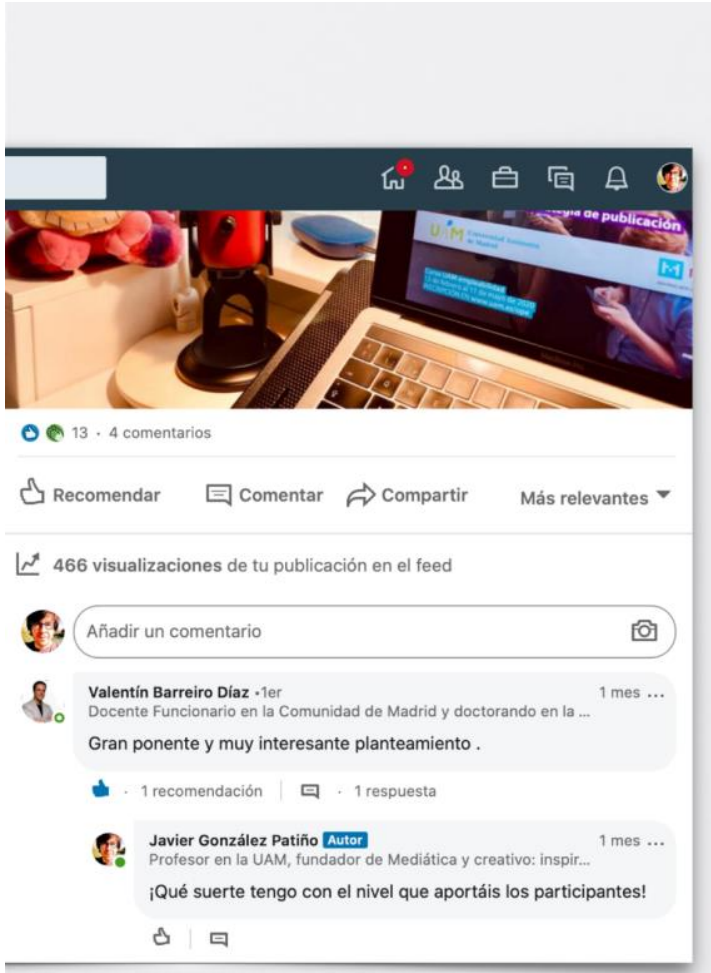

Figura 3. Participación abierta en diferentes plataformas de la web social

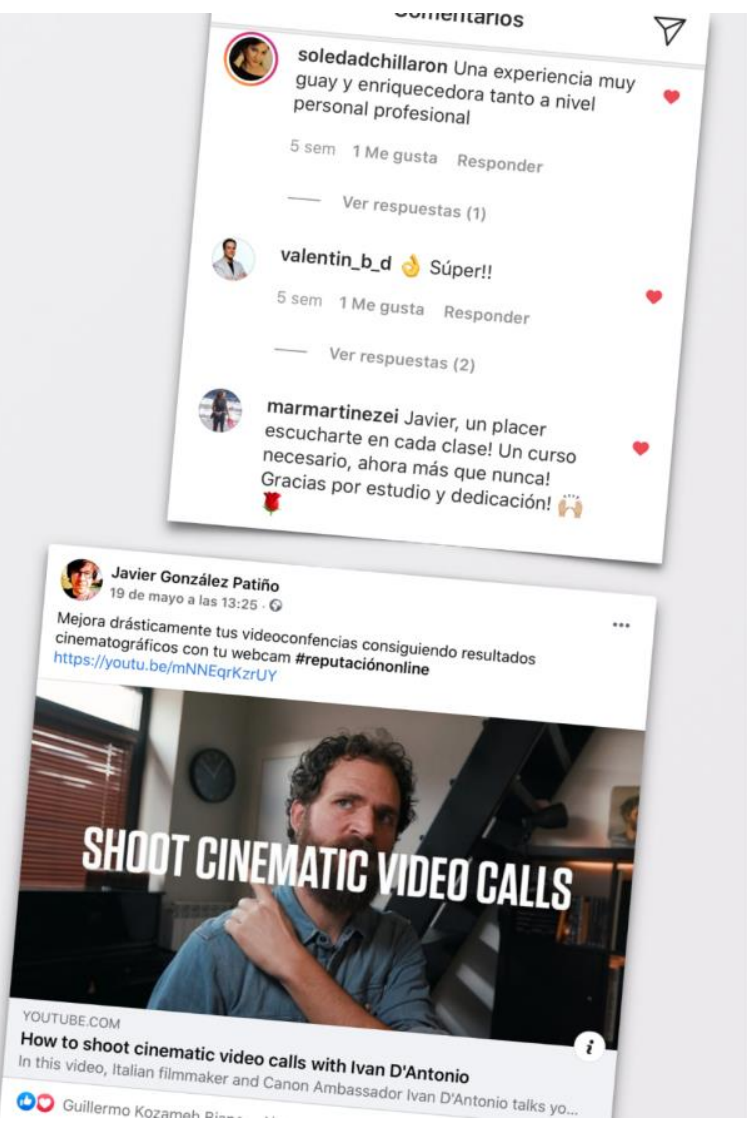

valentin_b_d S Súper! 1 Me gusta Responder (avier, un placer necesario, ahora clase! Un curso Gracias por estudio que nunca! 3 Javier González Patiñ Mejora drástica cinematográficos con tus videoconfencies consiguiendo resultad os

.

Se podrían resumir los objetivos de la comunidad de práctica fundada desde el interés de las y los participantes en torno a la creación y desarrollo de su propia identidad profesional en la web social como sigue: pretendemos estar presentes en aquellas plataformas del medio digital relevantes para participantes y posibles interesados en la gestión de la reputación online, escuchando y procesando información valiosa, potenciando la reflexión y el debate en torno a nuestra actividad, aportando herramientas que faciliten la participación y la reflexión más allá de la celebración de sesiones síncronas en remoto, y dinamizando la interacción mediante la promoción de una práctica participativa coherente con nuestro estilo e intereses como organizadores y colaboradores.

\section{El curso \#UAMskills de identidad digital como una práctica de educación personalizada.}

El diseño e implementación de la experiencia educativa sobre reputación online \#UAMskills permite identificar aquellas dimensiones o elementos de la personalización educativa descritos anteriormente (ver Figura 1) e identificados como relevantes para un planteamiento pedagógico propio de la actual sociedad conectada. Vamos a ilustrarlos mediante algunos ejemplos encontrados.

La transformación hacia experiencias expandidas en educación superior: curso \#UAMskills de identidad digital. Javier González-Patiño y Moisès Esteban-Guitart. 


\subsection{Identidades de los aprendices}

En lugar de preparar una guía didáctica centrada en una serie de contenidos esenciales que el aprendiz deba conocer sobre las redes sociales, se les ofreció una guía de participación en un programa orientado a una misión: crear su propia identidad digital. Precisamente la actividad con la que arrancaron la primera sesión síncrona fue realizar una búsqueda de sus rastros de presencia en la web social y para la segunda, una búsqueda de referencias visuales y discursivas que consideraran como inspiradoras para definir su posicionamiento como "marca personal" en internet, un paradigmático ejemplo de producción de un artefacto identitario que indague sobre los intereses y experiencias de las y los participantes, y que al ser prestando al resto en la segunda sesión, sirvió para configurar itinerarios de aprendizaje particulares que discurrieron en paralelo pero dando cohesión a la naciente comunidad de práctica que se pretendía promover en ese momento. La diversidad profesional del grupo es una constante en todas las ediciones del curso, que se ofrece a las y los estudiantes y titulados de todas las facultades de la UAM, y una de las claves para conseguir que funcione como una comunidad de práctica, orientada por el interés compartido en mejorar su competencia mediática. Por tanto, que los proyectos profesionales sean de ámbitos tan distintos (médicos, nutricionistas, administración de empresas, marketing, psicólogos o modelos profesionales, entre muchos otros) proporciona la oportunidad de transferir prácticas o inspiración mediante mecanismos de liderazgo distribuido legitimado por las y los participantes y en los que el profesor acompaña desde un posicionamiento de mediación-facilitación. En futuras ediciones del curso se planteará incluso la utilización de la aplicación Flipgrid de Microsoft (https://info.flipgrid.com) que pone en primer plano lo que aportan las y los aprendices, mediante vídeos de corta duración, y con una funcionalidad de conexión con otros grupos ampliando así las posibilidades de realizar colaboraciones abiertas.

\subsection{Procesos de reflexión}

Una de las actividades a las que se dedicó más tiempo durante las sesiones síncronas en remoto a lo largo del curso, fue la de presentar individualmente los progresos en la misión de cada participante para recibir retroalimentación por parte del resto del grupo. Además de promover la participación convirtiendo cada videoconferencia en un evento realmente interactivo, aspecto que sorprendió a las y los estudiantes por su dinamismo ya que esperaban sesiones de exposición teórica en formato "busto parlante" como era habitual en otras asignaturas y cursos, la reflexión entre iguales fue especialmente valorada por la oportunidad de situar los aprendizajes que estaban llevando a cabo, individual y colectivamente, en relación con sus intereses, objetivos y elecciones. Incorporando sus enfoques y perspectivas en un proceso continuo de auto y heteroevaluación (ver Figura 4), pues la intención era plantear una propuesta basada en la autoregulación metacognitiva (Sáiz-Manzanares y Queiruga-Dios, 2017), nos centramos en la dimensión reflexiva y de proyección transformadora respecto a la utilidad de los resultados de aprendizaje.

La transformación hacia experiencias expandidas en educación superior: curso \#UAMskills de identidad digital. Javier González-Patiño y Moisès Esteban-Guitart. 


\section{CRITERIO}

\section{Sesiones síncronas}

\section{Evaluación continua}

- Series individuales de material audiovisual (investigación), referencias compartidas y otras aportaciones inspiracionales.

- Toolkit o memoria de estilo digital.

- Tutoría.

- Intervenciones sobre el trabajo de otros participantes.

\section{Presentación final}

- Presentación de resultados ante el resto de participantes.

- Autoevaluación (rúbricas).

TOTAL

\section{PONDERACIÓN}

Requisito
10

20

5

15

20

30

$100 \%$

Figura 4. Procedimiento de evaluación incluido en la guía de participación

\subsection{Agencia del aprendiz}

Las y los estudiantes eran partícipes del control sobre sus aprendizajes no solo respecto a la evaluación, como acabamos de ver, sino en la definición de objetivos, se trata de un programa orientado a una misión en la que crean su propia caja de herramientas (toolkit) para definir su posicionamiento o identidad profesional digital, y en el codiseño de actividades, pues además de su participación en redes sociales, el contenido de las sesiones síncronas en remoto se negociaba en cada una de ellas y disponían de tiempo para talleres de "cacharreo" para configurar sus perfiles o utilizar herramientas y plataformas. De hecho, ni siquiera se les entregó el habitual documento de referencia de las asignaturas en educación superior, la guía docente, que define de forma reglamentaria entre otros los contenidos, objetivos, actividades y evaluación. En su lugar, mediante la guía de participación, se les invitó a construir una comunidad alrededor de sus intereses y objetivos (que indagaríamos a través de sus artefactos identitarios), decidiendo conjuntamente la organización de las actividades y creando conocimiento de manera distribuida y abierta.

\subsection{Itinerario de aprendizaje}

Cada miembro de esta comunidad se apropia de la experiencia expandida escogiendo y creando diferentes trayectorias tanto textuales como de participación. La apropiación y usos de los recursos y del espacio digital, tanto público como privado, es heterogénea y para cierta parte de las y los estudiantes supone un cambio profundo tanto en la manera de gestionar información y documentos como en la de crear conocimiento y compartirlo. No obstante, dado el modo en que lo expandido permite compilar, distribuir y almacenar

La transformación hacia experiencias expandidas en educación superior: curso \#UAMskills de identidad digital. Javier González-Patiño y Moisès Esteban-Guitart. 
los materiales generados participativamente en las sesiones síncronas en remoto, también encontramos interconexiones (lazos intertextuales, por ejemplo) entre las reflexiones compartidas que son bastante intensas.

El cronograma propuesto y que se observa en la Figura 2, permite identificar una organización que se alinea alrededor de unas sesiones síncronas en remoto, que podrían resultar clases en el equivalente presencial, y aunque plantean cierta estructura de actividades y contenidos conceptuales, en la práctica fueron negociados en función de los avances de cada participante en su proyecto personal, autentico centro de gravedad de los encuentros virtuales. Para flexibilizar aún más estos itinerarios, conviene recordar que los recursos disponibles eran compartidos a conveniencia del interés tanto en espacios de internet privados para el grupo (los mensajes de la lista de correo o la carpeta de Drive del curso) como en espacios públicos de la web social (en cualquier red o plataforma utilizando el hashtag \#reputaciononline para facilitar el seguimiento y recuperación). Esta narrativa transmedia, que refleja una diversidad de alternativas próximas a los usos y prácticas mediáticas de los participantes, permitió una apropiación muy personal (engagement) y fluida (seamless) de la experiencia de aprendizaje que las y los estudiantes reflejaron en las encuestas de satisfacción:

El curso, planteado en principio como presencial, ha tenido que reorientarse hacia online debido a las circunstancias del covid19. No veo por qué no pueda continuarse online. De hecho, parece más adecuado. En cuanto a organización y contenido, perfecto. El profesor conoce muy a fondo el contenido y por su competencia en distintas materias (psicología, comunicación, investigación, etc.), le hacen especialmente idóneo para liderarlo.

Extracto 2. Comentarios recogidos en las encuestas de satisfacción de las y los estudiantes

\subsection{Vinculación-conexión múltiple}

La red de interconexiones entre recursos, presentaciones, debates y reflexiones, más allá de los encuentros remotos, sobre la propia identidad digital, se despliegan a lo largo del curso dando forma a una experiencia de multimodalidad comunicativa orientada por el interés de los miembros de esta comunidad de práctica en construir su presencia profesional en redes sociales. A los contenidos que propone inicialmente el profesor hay que agregar las aportaciones públicas y privadas que comparten el resto conforme avanzan en su misión. En Classroom, LMS que funciona como nodo, se organiza la actividad incluyendo herramientas conjuntas de lista de correo, calendario, almacenamiento, propuesta de actividades y gestión de entregas, etc. Pero se diseñó para conectar el aprendizaje a nivel individual, así como, para articular la vinculación con otros espacios, como las redes sociales, otros agentes, como antiguos alumnos o invitados, y otras instituciones, como la colaboración con Mediática para el asesoramiento participativo de transformación digital (y que entre otros, permite este estudio) o con Hootsuite para disfrutar en paralelo y de manera optativa de su programa de educación superior Hootsuite's Student Program con certificación propia internacional. De manera complementaria, el espacio público digital de las redes sociales se ha incorporado como escenario para desarrollar y difundir la actividad, fortaleciendo la participación y ampliando las posibilidades de conversación independientemente de la ubicación geográfica y de lo temporal, permitiendo contar con formatos abiertos para continuar el aprendizaje participativo después de los encuentros síncronos y perdurar una vez pasados los mismos.

La transformación hacia experiencias expandidas en educación superior: curso \#UAMskills de identidad digital. Javier González-Patiño y Moisès Esteban-Guitart. 


\subsection{Acompañamiento}

El planteamiento operativo para dinamizar la participación consistió en transformar el rol del docente en el de moderador de comunidad virtual partiendo de la aproximación de las comunidades de práctica (Sanz, 2005). Esta moderación o mediación implica, entre otras, tutela, monitorización, gestión, seguimiento y dinamización, pues el moderador a través de una actitud proactiva cuenta entre sus funciones:

- Presentar, planificar y estimular la adhesión al marco de la guía de estilo de esta comunidad abierta.

- Monitorizar la red, no solo los perfiles que se habiliten, en busca de conversaciones sobre el área de interés de la comunidad.

- Inspirar, extraer lo relevante de la escucha y hacerlo circular entre los participantes.

- Orientar para mejorar la calidad de la participación aplicando los protocolos deseables para la comunidad (códigos de estilo, supervisión de folcsonomías o indexaciones sociales, relación con líderes de opinión, etc.).

- Identificar líderes, tanto interna como externamente a la comunidad, animando la participación o reclutando personas de cierta relevancia.

- Encontrar vías de colaboración y dinamizar la conversación entre los miembros.

- Resolver dudas, proponer ejemplos y aclaraciones adicionales.

La guía de participación que recibieron las y los estudiantes antes del inicio de curso destacaba explícitamente la importancia del acompañamiento, flexibilidad y relevancia sobre lo instruccional, en una sección de garantías de calidad que incluía los siguientes criterios: control de participación, tutorías y acompañamiento, valoración de la calidad docente percibida por el alumno (mediante encuestas de valoración del interés del curso y de valoración del trabajo del profesor) y autoevaluación del director de curso.

\section{Discusión y conclusiones}

Más allá del efecto de urgencia provocado por la situación de confinamiento forzoso generalizado (Bozkurt y Sharma, 2020), los retos que tiene que afrontar la educación en nuestra sociedad de la cultura digital ya situaban a la investigación en una posición crítica, vivimos un momento histórico de transformación tan rápida y profunda que exige a los profesionales de la enseñanza encontrar soluciones para una sociedad que necesita restablecer cierto equilibrio entre incertidumbre y posibilidades (Akama et al., 2018; Iglesias, 2020). En un futuro próximo probablemente el rol del profesor estará más próximo al de un profesional con un elevado grado de autogestión al que se requerirán competencias propias de un científico social (Kalantzis y Cope, 2012).

A través de la descripción e ilustración de esta experiencia de aprendizaje expandido se ha tratado de entender las lógicas de lo digital como una forma de estar y dar sentido al mundo en el que vivimos (Horst y Miller, 2012) donde los medios permiten conectar prácticas a través del tiempo y en diferentes escenarios para propiciar aprendizajes situados, conectados y dirigidos por el interés (Esteban-Guitart et al., 2020; GonzálezPatiño y Esteban-Guitart, 2019). Además, se señalan y comparten ciertas cuestiones

La transformación hacia experiencias expandidas en educación superior: curso \#UAMskills de identidad digital. Javier González-Patiño y Moisès Esteban-Guitart. 
metodológicas con la intención de ayudar a intervenir o inspirar para la mejora en procesos de cambio educativo, a la vez que se genera conocimiento científico desde una perspectiva participativa y transdisciplinar característica de nuestra sociedad conectada. Una búsqueda que se mueve en el intersticio de contextos reconocibles (sean estos institucionalizados, formales o no) expandidos por otros espacios públicos y privados digitales con estructuras participativas complejas y dinámicas que en lugar de imponerse se consensuan (González-Patiño, 2018).

Pero, por otro lado, necesitamos identificar posibles barreras a la colaboración que emergen incluso dentro de las formas más sólidas de cultura participativa. Es posible que las personas involucradas en una comunidad hayan naturalizado como universales ciertas prácticas que pueden convertirse en grandes obstáculos para alguien que no está tan involucrado culturalmente. En este estudio de caso, nos estaríamos refiriendo precisamente a los usos y prácticas digitales. Las personas que deciden unirse a una comunidad que comparte un interés determinado, han podido tener previamente diferentes grados de acceso a ciertos materiales o prácticas. Esta reflexión, que nos llevaría a analizar posibles barreras a la participación por apropiarnos del espacio digital de internet, estaría en línea con las propuestas que plantea el programa de fondos digitales de identidad (González-Patiño y Esteban-Guitart, 2015) que promueve la indagación de los fondos culturales (artefactos o instrumentos culturales) y usos relevantes e intereses mediados por lo digital, con la intención de incorporarlos a las prácticas de aprendizaje o de participación y así convertirlas en un proceso significativo y sensible a las formas de vida de los participantes. Y este precisamente, es el principio sobre el que se construye una actuación educativa basada en la personalización. Las personas pueden ser alentadas o desalentadas a desplegar sus identidades, a expresar ciertos intereses o deseos, y tendrán más o menos probabilidades de incorporarse a determinadas comunidades de práctica. Esas comunidades pueden ser abiertas y, al mismo tiempo, limitar la participación porque el interés en torno al cual se organizan sea más fácil de encontrar en función de ciertos antecedentes culturales y socioeconómicos. Aunque de manera excepcional, un estudiante inicialmente inscrito para realizar el curso de reputación online en modo presencial decidió abandonar tras ser adaptado a una versión en remoto y mediada digitalmente, sin ni siquiera animarse a probar:

Me apunté a este curso porque la docencia era presencial y el nuevo entorno de docencia lo aborrezco totalmente [...]. Y lo afirmo tras haber gestionado proyectos dispersos en ámbito internacional, donde las retro-tecnologías de la_no comunicación son vitales cuan menos para entender lo que cada uno quiere y hacer o no lo que uno espera.

Extracto 3. Comentarios recogidos en las e ncuestas de satisfacción de las y los estudiantes

En la sociedad red (Castells, 2009) necesitamos establecer estructuras red, locales y globales, de colaboración abierta entre sociedad, investigación, educación e innovación en ecosistemas que, con una perspectiva de cambio para la mejora social, promuevan la creación de experiencias de enseñanza y aprendizaje situadas en nuevos contextos, superando los modelos tradicionales basados en la instrucción entendida como mera transmisión de contenidos (Álvarez-Arregui, 2019). Avanzar en esta exploración permitirá dejar atrás definitivamente el debate propio del anterior siglo, el XX, acerca de los opuestos entre aprendizaje presencial-offline y virtual-online, incorporando experiencias y trayectorias de aprendizaje como marco para la nueva actuación educativa (Esteban-Guitart et al., 2018; Gee y Esteban-Guitart, 2019).

La transformación hacia experiencias expandidas en educación superior: curso \#UAMskills de identidad digital. Javier González-Patiño y Moisès Esteban-Guitart. 
Los nuevos modelos pedagógicos tienen que estar preparados para hacer frente a escenarios de elevada complejidad e incertidumbre (Iglesias et al., 2020) dando soporte, mediante evidencias, para desarrollar experiencias expandidas, flexibles y distribuidas en torno a una narrativa transmedia a través de la cual profesores y estudiantes naturalicen la cultura digital en lo que a oportunidades y procesos de aprendizaje se refiere (GonzálezMartínez et al., 2019). Concretamente en el contexto de la educación superior, la personalización educativa, que no pretende ser un mero proceso de individualización (customización), persigue incorporar la libertad, control y responsabilidad (agencia) de los aprendices, haciéndoles partícipes de la coordinación de los procesos de aprendizaje para convertirlos en proyectos o misiones personales que facilitarían aprendizajes con sentido y valor personal (Coll, 2018; Coll, Esteban-Guitart e Iglesias, 2020; Jubany, 2012). Como consecuencia, el rol docente tendría que orientarse hacia la mediación y acompañamiento (ayuda, orientación, escucha) trascendiendo los tradicionales vínculos instrumentales o más funcionales (Pérez Guerrero y Ahedo Ruiz, 2020; Tarabini, 2020).

Presentación del artículo: 25 de octubre de 2020 Fecha de aprobación: 10 de diciembre de 2020 Fecha de publicación: 08 de enero de 2021

González-Patiño, J. y Esteban-Guitart, M. (2021). La transformación hacia experiencias expandidas en educación superior: curso \#UAMskills de identidad digital. RED. Revista de Educación a Distancia, 21(65). https://doi.org/10.6018/red.433881

\section{Financiación}

Este trabajo forma parte de una investigación realizada en el marco del proyecto EDU2017-83363-R: "Inclusión y Mejora del Aprendizaje a través de la Contextualización Educativa. Avances en la aproximación de los Fondos de conocimiento e identidad (IMACE)". Este proyecto está financiado por el Plan Nacional de Investigación del Gobierno de España.

\section{Referencias bibliográficas}

Akama, Y., Pink, S., y Sumartojo, S. (2018). Uncertainty and Possibility. New Approaches to Future Making in Design Anthropology. Routledge.

Álvarez-Arregui, E. (2019). Evolución de la Universidad en la Sociedad del Aprendizaje y la Enseñanza. El valor de las competencias en el desarrollo profesional y personal. Aula Abierta, 34(4), 349-372. https://doi.org/10.17811/rifie.48.4.2019.349-372

Álvarez-Arregui, E., y Arreguit, X. (2019). El futuro de la Universidad y la Universidad del Futuro. Ecosistemas de formación continua para una sociedad de aprendizaje y enseñanza sostenible y responsable. Aula Abierta, 48(4), 447-480. https://doi.org/10.17811/rifie.48.4.2019.447-480

Barron, B. (2006). Interest and Self-Sustained Learning as Catalysts of Development: A Learning Ecology Perspective. Human Development, 49(4), 193-224. https://doi.org/10.1159/000094368

La transformación hacia experiencias expandidas en educación superior: curso \#UAMskills de identidad digital. Javier González-Patiño y Moisès Esteban-Guitart. 
Bozkurt, A., y Sharma, R. C. (2020). Emergency remote teaching in a time of global crisis due to CoronaVirus pandemic. Asian Journal of Distance Education, 15(1), 1-6. https://doi.org/10.5281/zenodo.3778083

Bronfenbrenner, U. (1985). Contexts of child rearing problems and prospects. Journal for the Study of Education and Development, 8(29), 45-55. https://doi.org/10.1080/02103702.1985.10822058

Bronfenbrenner, U. (1987). La ecología del desarrollo humano. Experimentos en entornos naturales y diseñados. Paidós.

Bruner, J. S., y Palacios, J. (2008). Desarrollo cognitivo y educación (6. ${ }^{\mathrm{a}}$ ed.). Morata.

Castells, M. (2009). Comunicación y poder. Alianza.

Chen, C. M. (2008). Intelligent web-based learning system with personalized learning path guidance. Computers y Education, 51(2), 787-814. https://doi.org/10.1016/j.compedu.2007.08.004

Coll, C. (2013). El currículo escolar en el marco de la nueva ecología del aprendizaje. Aula de Innovación Educativa, 219, 31-36.

Coll, C. (2018). La personalización del aprendizaje escolar, una exigencia de la nueva ecología del aprendizaje. Dosier Graó: La personalización del aprendizaje, 3, 511.

Coll, C., Esteban-Guitart, M., e Iglesias, E. (2020). Aprendizaje con sentido y valor personal. Estrategias, recursos y experiencias de personalización educativa. Graó.

DiGiacomo, D. K., Van Horne, K., Van Steenis, E., y Penuel, W. R. (2018). The material and social constitution of interest. Learning, Culture and Social Interaction, 19, 51-60. https://doi.org/10.1016/j.lcsi.2018.04.010

Esteban-Guitart, M. (2016). Funds of Identity. Connecting Meaningful Learning Experiences in and out of School. Cambridge University Press. https://doi.org/10.1017/CBO9781316544884

Esteban-Guitart, M. (2019). Identity in education and education in identities: Connecting curriculum and school practice to students' lives and identities. En P. Hviid y M. Martsin (Eds.), Culture in education and education in culture (pp. 159-175). Springer.

Esteban-Guitart, M., Coll, C., y Penuel, W. R. (2018). Learning across Settings and Time in the Digital Age. Digital Education Review, 33, 1-16. https://doi.org/10.1344/der.2018.33.\%p

Esteban-Guitart, M., y Gee, J. P. (2020). "Inside the head and out in the world". An approach to deep teaching and learning. Multidisciplinary Journal of Educational Research, 10, 1-25. https://doi.org/10.4471/remie.2020.4868

Esteban-Guitart, M., y Moll, L. C. (2014). Funds of Identity: A new concept based on the Funds of Knowledge approach: Culture y Psychology, 20(1), 31-48. https://doi.org/10.1177/1354067X13515934

Esteban-Guitart, M., Monreal-Bosch, P., Palma, M. y González-Ceballos, I. (2020). Sustaining Students' Identities within the Context of Participatory Culture.

La transformación hacia experiencias expandidas en educación superior: curso \#UAMskills de identidad digital. Javier González-Patiño y Moisès Esteban-Guitart. 
Designing, Implementing and Evaluating an Interactive Learning Activity. Sustainability, 12(12), 4870. https://doi.org/10.3390/su12124870

Esteban-Guitart, M., DiGiacomo, D. K., Penuel, W. R. y Ito, M. (2020). Principios, aplicaciones y retos del aprendizaje conectado. Contextos Educativos, 26, 157176. https://doi.org/10.18172/con.3966

Esteban-Guitart, M., Serra, J. M., y Vila, I. (2017). Informationalism and informalization of learnings in 21 st century. A qualitative study on meaningful learning experiences. Social and Education History, 6(1), 1-25. https://doi.org/10.17583/hse.2017.2111

Esteban-Guitart, M., DiGiacomo, D., Penuel, W. y Ito, M. (2020). Principios, aplicaciones y retos del aprendizaje conectado. Contextos Educativos, 26, 157176. https://doi.org/10.18172/con.3966

Gee, J. P. (2010). New digital media and learning as an emerging area and «worked examples» as one way forward. The MIT Press.

Gee, J. P. (2013). The anti-education era: Creating smarter students through digital learning. Palgrave Macmillan.

Gee, J. P., y Esteban-Guitart, M. (2019). Designing for deep learning in the context of digital and social media. [El diseño para el aprendizaje profundo en los medios de comunicación sociales y digitales]. Comunicar, 58, 9-18. https://doi.org/10.3916/C58-2019-01

Giani, M., y Fox, H. L. (2017). Do stackable credentials reinforce stratification or promote upward mobility? An analysis of health professions pathways reform in a community college consortium. Journal of Vocational Education y Training, 69(1), 100-122. https://doi.org/10.1080/13636820.2016.1238837

González-Martínez, J., Esteban-Guitart, M., Rostan-Sanchez, C., Serrat-Sellabona, E., y Estebanell-Minguell, M. (2019). What's up with transmedia and education? A literature review. Digital Education Review, 36, 207-222. https://doi.org/10.1344/der.2019.36.207-222

González-Patiño, J. (2011). Rutinas de la infancia urbana mediadas por la tecnología: Un análisis visual. Papeles de Trabajo sobre Cultura, Educación y Desarrollo Humano, 7(2), 1-16.

González-Patiño, J. (2012). Mediática | Inspiring the new learning for the digital culture. https://mediatica.co/

González-Patiño, J. (2018). Learn, Teach and Share, Participation in Expanded Educational Communities: Madrid Pikler Seminar as a Practice of Continuing Education between Childhood Professionals. Digital Education Review, 33, 203216. https://doi.org/10.1344/der.2018.33.203-216

González-Patiño, J., y Esteban-Guitart, M. (2014). Some of the challenges and experiences of formal education in a Mobile-Centric Society (MCS). Digital Education Review, 25, 64-86. https://doi.org/10.1344/der.2014.25.64-86

González-Patiño, J., y Esteban-Guitart, M. (2015). Fondos digitales de conocimiento e identidad: Un análisis etnográfico y visual. Papeles de Trabajo sobre Cultura, Educación y Desarrollo Humano, 11(2), 20-25.

La transformación hacia experiencias expandidas en educación superior: curso \#UAMskills de identidad digital. Javier González-Patiño y Moisès Esteban-Guitart. 
González-Patiño, J., y Esteban-Guitart, M. (2019). Affinity online: How connection and shared interest fuel learning. Mind, Culture, and Activity, 26(4), 383-386. https://doi.org/10.1080/10749039.2019.1680695

Grant, P., y Basye, D. (2014). Personalized learning: A guide for engaging students with technology. International Society for Technology in Education.

Horst, H. A., y Miller, D. (2012). Digital anthropology. Berg.

Iglesias, E., González-Patiño, J., Lalueza, J. L., y Esteban-Guitart, M. (2020). Por una educación crítica, intergeneracional, sostenible y comunitaria. Manifiesto en tiempos de pandemia. Revista Internacional de Educación para la Justicia Social (RIEJS), 9(3), 181-198. https://doi.org/10.15366/riejs2020.9.3.010

Ito, M., Gutiérrez, K., Livingstone, S. M., Penuel, B., Rhodes, J., Salen, K., Schor, J., Sefton-Green, J., y Watkins, S. C. (2013). Connected Learning: An Agenda for Research and Design. Digital Media and Learning Research Hub.

Jenkins, H. (2008). Convergence culture: La cultura de la convergencia de los medios de comunicación. Paidós.

Jenkins, H., Ford, S., y Green, J. (2013). Spreadable media: Creating value and meaning in a networked culture. New York University Press.

Jenkins, H., Ito, M., y Boyd, D. (2015). Participatory culture in a networked era: A conversation on youth, learning, commerce, and politics. Polity Press.

Jovés, C., Siqués, C. y Esteban-Guitart, M. (2015). The incorporation of funds of knowledge and funds of identity of students and their families into educational practice. A case study from Catalonia, Spain. Teaching and Teacher Education, 49, 68-77. https://doi.org/10.1016/j.tate.2015.03.001

Jubany, J. (2012). Aprendizaje social y personalizado: Conectarse para aprender. Editorial UOC. https://www.editorialuoc.cat/aprendizaje-social-y-personalizadoconectarse-para-aprender

Kalantzis, M., y Cope, B. (2012). New learning: Elements of a science of education (2. ${ }^{\mathrm{a}}$ ed.). Cambridge University Press.

Lacasa, P. (2018). Expresiones del futuro. Cómo se comunicarán las próximas generaciones. Morata.

Lacasa, P., de la Fuente, J. y Méndez, L. (2020). Methodological challenges for digital ethnographers. Reflecting on teenager practices mediated by mobile devices. Papeles de Trabajo sobre Cultura, Educación y Desarrollo Humano, 16(1), 1-17.

Lacasa, P., de la Fuente, J., Garcia-Pernia, M. y Cortés, S. (2017). Teenagers, fandom and identity. Persona Studies, 3(2), 51-65.

Lafuente, A., Gómez, D., y Freire, J. (2018). El arte de documentar. Zenodo. https://doi.org/10.5281/zenodo.1195211

Lave, J., y Wenger, E. (1991). Situated learning: Legitimate peripheral participation. Cambridge University Press.

Minguillón, J., Mor, E., Santanach, F., y Guàrdia, L. (2005). Personalización del proceso de aprendizaje usando learning objects reutilizables. Revista de Educación a Distancia (RED), IV , 1-14.

La transformación hacia experiencias expandidas en educación superior: curso \#UAMskills de identidad digital. Javier González-Patiño y Moisès Esteban-Guitart. 
Mitchell, C. (2011). Doing visual research. Sage Publications.

Nasir, N. S., de Royston, M. M., Barron, B., Bell, P., Pea, R., Stevens, R., y Goldman, S. (2020). Learning pathways. En N. S. Nasir, C. D. Lee, R. Pea, y M. M. de Royston (Eds.), Handbook of the Cultural Foundations of Learning (pp. 201-218). Routledge.

Oliveira, A. M. C., y Coelho, C. M. M. (2020). Subjective development process as a path to school learning: The classroom as a dialogic relational context. Studies in Psychology, 41(1), 115-137. https://doi.org/10.1080/02109395.2019.1710803

Penuel, W. R., y Johnson, R. (2016). Review of Continued Progress: Promising Evidence on Personalized Learning. National Education Policy Center. https://nepc.colorado.edu/thinktank/review-personalized-learning

Pereira, S., Fillol, J., y Moura, P. (2019). Young people learning from digital media outside of school: The informal meets the formal. [El aprendizaje de los jóvenes con medios digitales fuera de la escuela: De lo informal a lo formal]. Comunicar, 58, 41-50. https://doi.org/10.3916/C58-2019-04

Pérez Guerrero, J., y Ahedo Ruiz, J. (2020). La educación personalizada según García Hoz. Revista Complutense de Educación, 31(2), 153-161. https://doi.org/10.5209/rced.61992

Perin, D. (2011). Facilitating Student Learning Through Contextualization: A Review of Evidence. Community College Review, 39(3), 268-295. https://doi.org/10.1177/0091552111416227

Pink, S. (2013). Doing visual ethnography: Images, media, and representation in research $\left(3^{\mathrm{a}}\right)$. SAGE.

Rodríguez-Ruiz, B., Álvarez-Blanco, L., Martínez-González, R. A., y Epstein, J. L. (2019). Presentación del número Relación entre Centros Educativos, Familias y Entidades Comunitarias. Aula Abierta, 48(1), 7-10. https://doi.org/10.17811/rifie.48.1.2019

Sáiz-Manzanares, M. C., y Queiruga-Dios, M. Á. (2017). Evaluación de estrategias metacognitivas: Aplicación de métodos online. Revista de Psicología y Educación - Journal of Psychology and Education, 13(1), 23. https://doi.org/10.23923/rpye2018.01.156

Sanz, S. (2005). Gestión de comunidades de práctica virtuales: Acceso y uso de contenidos. RUSC. Universities and Knowledge Society Journal, 2(2), 26-35. https://doi.org/10.7238/rusc.v2i2.259

Staus, N. L., Falk, J. H., Penuel, W., Dierking, L., Wyld, J., y Bailey, D. (2020). Interested, Disinterested, or Neutral: Exploring STEM Interest Profiles and Pathways in A Low-Income Urban Community. Eurasia Journal of Mathematics, Science and Technology Education, 16(6), em1853. https://doi.org/10.29333/ejmste/7927

Tarabini, A. (2020). ¿Para qué sirve la escuela? Reflexiones sociológicas en tiempos de pandemia global. Revista de Sociología de la Educación-RASE, 13(2), 145-155. https://doi.org/10.7203/RASE.13.2.17135

Thomson, P. (2010). Doing visual research with children and young people. Routledge.

La transformación hacia experiencias expandidas en educación superior: curso \#UAMskills de identidad digital. Javier González-Patiño y Moisès Esteban-Guitart. 
Van Dijck, J. (2013). The culture of conectivity: A critical history of social media. Oxford University Press.

Walkington, C., y Hayata, C. A. (2017). Designing learning personalized to students' interests: Balancing rich experiences with mathematical goals. ZDM, 49(4), 519530. https://doi.org/10.1007/s11858-017-0842-z

Wenger, E. (2005). Communities of practice: Learning, meaning, and identity. Cambridge University Press.

Wenger, E., McDermott, R., y Snyder, W. (2002). Cultivating communities of practice: A guide to managing knowledge. Harvard Business School Press. 